\title{
Territorial gain dynamics regulates success in attacking sub-phases of team sports
}

\author{
Vanda Correia $^{\mathrm{a}, *}$, Duarte Araújo ${ }^{\mathrm{a}}$, Keith Davids ${ }^{\mathrm{b}}$, Orlando Fernandes ${ }^{\mathrm{c}}$, Sofia Fonseca ${ }^{\mathrm{d}}$ \\ ${ }^{a}$ Faculty of Human Kinetics, Technical University of Lisbon, Estrada da Costa, 1495-688 Cruz Quebrada, Portugal \\ ${ }^{\mathrm{b}}$ School of Human Movement Studies, Queensland University of Technology, Australia \\ ${ }^{\mathrm{c}}$ University of Évora, Portugal \\ d Lusófona University of Humanities and Technologies, Portugal
}

\section{A R T I C L E I N F O}

\section{Article history:}

Received 12 August 2010

Received in revised form

27 May 2011

Accepted 1 June 2011

Available online 15 June 2011

\section{Keywords:}

Pattern forming dynamics

Coordination variable

Decision making

Team sports

\begin{abstract}
A B S T R A C T
Background and objective: Field invasion games, such as rugby union, can be conceptualised as dynamic social systems in which the agents continuously interact to contest ball possession and territorial gain. Accordingly, this study aimed to identify the collective system dynamics of rugby union phases-of-play near the try line by investigating whether ball displacement trajectory on the playing field provides insights on successful team performance.

Methods: Five rugby union matches were videotaped involving teams at a national league performance level. From these matches, 22 second phases-of-play were selected and digitized for analysis. The variable "distance gained" was investigated as a potential coordination variable describing functional coordination between players and teams. This variable concerned the distance between ball initial position and ball current position over time and was used to define the degree of territory gained by an attacking team. Results: Analysis of distance gained dynamics in attacking sub-phases demonstrated the intermittent character of rugby union performers displacement trajectories on the playing field. Amplitude of ball movements was revealed as a distinguishing feature related to attacking effectiveness. Successful attacking phases displayed lower distances of positional retreat, with the maximum retreat distance achieved sooner in successful compared to unsuccessful phases-of-play. Autocorrelation and ApEn analyses suggested low system variability within time series data concerning both performance outcomes. However, evidence of less regularity and more complexity was found in unsuccessful phases-of-play. Conclusion: Results suggested that distance gained dynamics manifests a characteristic collective behaviour pattern that captures the macroscopic functional order of multi-player attack-defence systems in team sports like rugby union.
\end{abstract}

(c) 2011 Elsevier Ltd. All rights reserved.
Field invasion games can be characterised as complex, social neurobiological systems in which agents interact continuously to contest ball possession and seek territorial advantage. In the team sport of rugby union, for example, Greenwood (2003) proposed that "possession is only a potential advantage" (p. 20). The team in possession of the ball strives to maintain continuity of possession and to advance up field and score points. Conversely, opponents contest ball possession, hamper displacement of players with the ball, and attempt to advance up field to reach a position advantageous for a potential counter-attack. Within these player-environment interactions, the balance of contestability and continuity, coordination and competition, coexist in a complementary way and are believed to

\footnotetext{
* Corresponding author. Tel.: +351 214149209; fax: +351 214144712.

E-mail addresses: advcorreia@fmh.utl.pt, correia.vanda@gmail.com (V. Correia), daraujo@fmh.utl.pt (D. Araújo), k.davids@qut.edu.au (K. Davids), orlandoj@uevora.pt (O. Fernandes), sofiaccfonseca@gmail.com (S. Fonseca).
}

be depicted in team's displacement trajectory during performance. This perspective brings about the need to describe and explain the pattern forming dynamics of system agents over time (Passos, Araújo, Davids, \& Shuttleworth, 2008). In rugby union, a chief task constraint on decision making and actions of players is the backward pass (International Rugby Board, 2010). Due to this task constraint of the game negative team momentum (i.e., negative values of distance gained corresponding to a retreating movement of the ball) might be expected, hampering territorial gains by an attacking team. This influential rule of rugby union implies that the forward movement (territorial gain) of an attacking team is characterised by cycles of passing the ball, running with it to gain ground and being tackled. These cycles correspond to phases-of-play. A first phase-of-play or set play constitutes all restarts that follow a scrum, a lineout, a dropout or a free kick. A second phase-of-play constitutes all open play "where the team controlling the ball will aim to "recycle" the ball after a tackle contact is made and a ruck/maul (breakdown) occurs" (Best, McIntosh \& Savage, 2005, p. 816) (for a detailed description of 
game stoppages see the International Rugby Board manual, 2010). This study aimed to develop an understanding of collective decisional behaviour in the team sport of rugby union during attacking second phases-of-play. The specific objective was to understand whether the variable, distance gained, conveys the dynamics of decision making behaviours of attacking players during rugby union performance.

In social neurobiological systems such as team sports, patterns of behaviour emerge during performance from individual-environment goal-directed interactions (from 1-vs-1 to 15-vs-15 game subphases) and can be studied through attacker-defender symmetries and symmetry breakings, decisions and actions (Araújo, Davids, \& Hristovski, 2006). Team sports can be regarded as social neurobiological systems in a complementary perspective that integrates each performer's central nervous system, body-environment interactions (biological - or interactions of a biological movement system and its environment) in an interpersonal social system (Davids, Araújo, Shuttleworth, \& Button, 2003; Keil, Holmes, Bennett, Davids, \& Smith, 2000; Kelso, 2009; Neisser, 1994; Newell, 1986; Van Gelder \& Port, 1995). Social neurobiological systems are important to study because movement behaviour of each individual performer may be considered an emergent property of the continuous interactions of biological animated systems and the environment in goal-directed behaviours (Davids, Araújo, Shuttleworth, \& Button, 2003; Davids, Button, Araújo, Renshaw, \& Hristovski, 2006; Kelso, 2009; Newell, 1986). In social neurobiological systems, a relevant concept to understand is self-organization (Kelso, 1995, 2009). Self-organization implies that spatio-temporal patterns between system agents are not externally planned or organized by a coordinating agent according to a pre-existing template or a group internal representation, but rather are emergent from the non-linear interactions of the dynamic elements of the system (Schweitzer, 1997). As argued by Kelso (2009), emergent self-organizing dynamics in living systems (e.g., a team game), has its basis in information which is displayed through the parameters acting on the relevant variables of the system. Unlike inanimate systems, functional coordination underlies animated systems and within these systems information simultaneously acts and is originated from self-organizing processes (Kelso, 2009). Besides, functional coordination implies that agents in these systems exhibit a kind of spatio-temporal ordering that adapt in a flexible manner to varying circumstances (Kelso, 2002). An important variable which describes emergent, self-organized dynamic patterns in complex systems is the order parameter or collective variable (Kelso, 1995, 2009). Coordination variables are context-dependent and describe how patterns are formed and evolve across time in social neurobiological systems. They capture the functional organization among interacting elements and processes in space and time (Kelso, 2009). A coordination variable results from the cooperation between parts of a system and simultaneously constrains the behaviour of those individual parts (Kelso, 1995). In the performance context of competitive team sports, McGarry and colleagues (McGarry, Anderson, Wallace, Hughes, \& Franks, 2002) have viewed this variable as a measure of the relationship between individual system components.

In the current study, performance in rugby union sub-phases of attackers and defenders was analyzed to generalise towards collective decisional behaviours in team sports.

\section{Dynamics of territorial advantage in rugby union second phases-of-play}

Rugby union is a field invasion team game characterised by intermittent ('stop-go') patterns of play (e.g., Greenwood, 2003; Reed \& Hughes, 2006). Between recurrent game stoppages, each team in possession of the ball aims to gain ground relative to an initial starting position. A projected line parallel to the try line that identifies a gained position (i.e., where the ball was regained from the opposite team) is labelled the gain line and is a reference to measure the degree of territory successfully gained in an attack (Bessa, 2005; Greenwood, 2003). Another important performance measure exists in the contact line. This is another projected line parallel to the try line that identifies the current position of the ball. Since in rugby union the ball-carrier can be stopped by defenders by being tackled to the ground, the ball's initial position ("gain line") assessed against its current position ("contact line") defines the degree of territory gained by an attacking team. In this study we have hypothesized that "contact line" movement over time could capture the functional ordering of attacking and defending player synergies in a phase-of-play of a rugby union match. Synergies at this juncture are understood as the functional alignment of players who are constrained to act as a single coherent sub-unit (cf. Kelso, 2009) coordinating and competing to assist common performance goals. As a candidate variable to capture interpersonal coordination of attacking players in this team sport we investigated the distance between the gain line and the contact line. We assumed that ball displacement trajectory on the playing field might yield information about success in attacking phases-of-play in a rugby union match. In a previous analysis of neurobiological system coordination, Vereijken and colleagues (Vereijken, van Emmerik, Bongaardt, Beek, \& Newell, 1997) analyzed the dynamics of a single variable (i.e., centre of mass) regarding it as an encompassing measure to reflect the global dynamics of motor system degrees of freedom during a dynamic balancing task. Using similar experimental logic, in the present study we assumed that distance gained might be a variable capable of capturing the collective actions of the agents (e.g., players) in social neurobiological systems exemplified by rugby union matches.

Our aim was to identify the collective dynamics of attack-defence sub-phases of play that, due to proximity to the try line, were assumed to precede the possibility of a try being scored. To achieve this aim, analysis of the pattern dynamics of ball displacement trajectory was undertaken within phases-of-play in which the attacking team was playing inside the opponent's half of the field. Specifically, the focus was on the dynamics of progression and retreat of the ball in the field within second phases-of-play of rugby union performance.

\section{Method}

Sample

We investigated twenty two attack-defence rugby union second phases-of-play near the try line $\left(M_{\text {distance to the try line }}=\right.$ 21.39; $\mathrm{SD}=6.86 \mathrm{~m})$ recorded from five national rugby union senior league games (semi-finals and final of the Portuguese Rugby Union First League Championship 2007/08, and semi-final and final of the Portuguese Rugby Union Cup 2008). Data acquisition processes were compliant with the university institution ethical guidelines. Players taking part in these games, and in the phases-of-play considered for analysis, averaged 28 years of age $(M=27.75$; $\mathrm{SD}=4.03$ years). The participant group was homogenous regarding rugby union playing experience $(M=17.5 ; \mathrm{SD}=4.69$ years $)$. It is important to note that, due to try line proximity, the performance situations were selected for analysis because they were considered to precede the possibility of a try being scored. Given that a try is scored only with the ball in hand, the greater proximity of the ball to the try line was assumed to reflect situations in which the attacking team was closer to achieve the performance goals. Being closer to opponents' in-goal area, players are like this closer to score 
whether by grounding the ball within this area (i.e., try) or by kicking (i.e., penalty try, conversion goal, penalty goal, or dropped goal; see IRB, 2010 for detailed information). A phase-of-play was regarded as the period of time between breakdowns in play (e.g., ruck/maul) in an attacking team's possession time, previously identified in the study of Reed and Hughes (2006).

\section{Recording procedures}

The phases-of-play were captured by a digital video camera mounted on tripods and placed in a transversal plane (about $45^{\circ}$ in relation to the pitch lateral line) and elevated (about $12 \mathrm{~m}$ high) level in relation to the performance field. No particular camera angle conditions were obeyed for these recordings since the selected digitization procedures did not require it. The selected recording angle covered at least the $22 \mathrm{~m}$ area (i.e., between the try line and the $22 \mathrm{~m}$ line) as shown in Fig. 1. The camera zooming rate was fixed in order to simplify the motion image processing (i.e., calibration procedures with TACTO 7.0 software; see Duarte et al., 2010, for software details). Video images were transferred to a computer via USB cable, using Windows Movie Maker software, and saved as wmv files.

\section{Data inclusion/exclusion criteria}

From the rugby union matches recorded performance footage was obtained for analysis. Inclusion and exclusion criteria for footage selection were established based on theoretical and methodological arguments. One of the essential criteria was to consider only filmed sequences of second-phase possession by an attacking team. First-phase possession is characterised by an organized set-up. In this phase all players are conventionally positioned (generally attacking teams establish depth and defending teams adopt a flat disposition facing the attack) at an established distance, which customarily leads to a zero forward momentum (Reed \& Hughes, 2006). In contrast, due to the more uncertain initial conditions, second-phase attacks can lead to either positive or negative territorial gains for an attacking team. Rarely will all the players of both teams be in expected playing positions and the depth of the attack, in particular, might be weakly established. Since this study purports to examine self-organized collective pattern dynamics in rugby union match situations, the greater unpredictability of second phase-of-play situations is more conducive for achieving this aim.

Three other criteria for selection of the performance situations for analysis included: (i) occurrence when the attacking team was playing inside the opposition half, i.e., situations preceding scoring try possibility; (ii) initiation with a set piece, as a ruck or maul, and progressed to more open play; and (iii) termination with one of the following: (a) the attacking team moving forward (carrying or kicking the ball), (b) the defence regaining ball possession, (c) a game stoppage due to penalty or (d), the ball being put out of the field. We considered that the attacking principles of the game might be best assessed by determining the starting location of the contact line in a set piece and then studying it's evolution towards open play as result of agent interactions.

\section{Grouping successful and unsuccessful situations}

Successful phases-of-play were considered to be those in which the attack progressed beyond the initial gain position (gain line) after a game stoppage (e.g., a ruck or maul), despite a first move backwards due to the distinctive rugby union rule that forbids the forward pass. Conversely, unsuccessful phases-of-play were regarded as all those that displayed the same backward movement first followed by a forward movement, with the final ball position (i.e., new game stoppage) being further from the try line than the original position where the attacking phase started.

\section{Data processing}

Image treatment was achieved by a dedicated software package TACTO 7.0 (Fernandes, Folgado, Duarte, \& Malta, 2010), with digitizing occurring at 25 frames per second. This software package allowed the extraction of $x$ and $y$ pixel coordinates of ball displacement along selected footages of phases-of-play. However, since this output does not correspond to real pitch area coordinates, the transformation of the digitized data to real coordinates (with reference to the pitch actual dimensions) was achieved through the Direct Linear Transformations method (DLT) using MATLAB 7.0
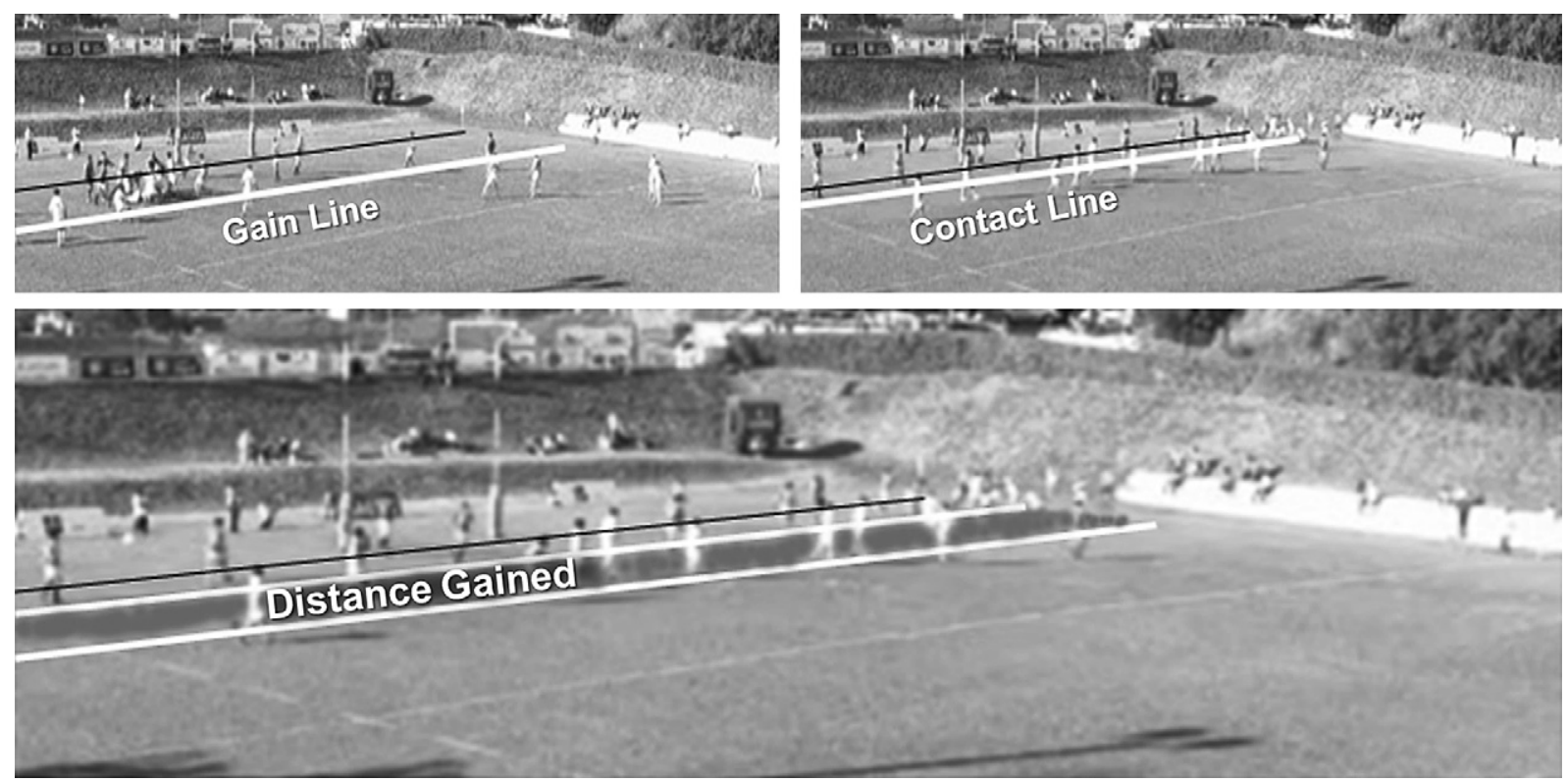

Fig. 1. Original game scene taken by the camera. Demarcation of the variable distance gained and the respective lines considered for its computation (illustrative scene). 
software. Using the same mathematical software, the time series positional data of ball displacement trajectories were obtained for each sample performance situation and analyzed to compute the variables of interest.

In order to capture the collective dynamics of rugby union second phases-of-play the first goal was to identify a potential coordination variable that could reflect the global dynamics of this performance sub-phase. The coordination variable selected for analysis was distance gained. Distance gained was calculated by the difference (in meters) between the ball's starting position in a developing attack (defining the "gain line") and its re-positioning over time (defining the "contact line"). The obtained value of this variable would be positive if the team with the ball progresses up the field in relation to its initial position, implying that the attacking team was successfully advancing to occupy opposition territory. On the other hand, the value would be negative when the ball retreated in relation to its initial gain position, implying a defensive advantage in territorial occupation dynamics. Time series of varying lengths were obtained from these computed distance data. Time series analysis methods were used to identify changes to the distance gained dependent variable in these performance situations based on analysis of ball displacement. This approach required the need to identify and describe pattern dynamics in the observed time series data.

A number of useful methods currently exist for qualitatively or quantitatively analyzing patterns in data from a single measured variable, or of the patterns of association between more than one variable over time (for reviews see Mullineaux, 2008; Mullineaux, Bartlett, \& Bennett, 2001). However, as underlined by Mullineaux et al. (2001) sometimes the patterned relationship between data is not obvious, nor linear. Thus, an alternative method for depicting data variability and to assess the pattern forming dynamics of data across performance trials is to plot one variable as a function of another (Mullineaux et al., 2001).

In order to describe the collective system dynamics and identify patterns of interaction between variables the analysis started by a portrayal of distance gained as a function of normalized time. Position-time graphs allowed visual checking for signs of stability/ and variability in that variable over time and for potential trends distinguishing between successful and unsuccessful team performance (further confirmed by means of a Linear Discriminant Analysis (LDA) performed in Matlab R2008). In addition the structural characteristics of the time series data were verified by means of state space reconstruction (Stergiou, Buzzi, Kurz, \& Heidel, 2004). For this purpose a two-dimensional phase plot, with distance gained $D$ (in meters) against its first derivate $D^{\prime}$ (in meters per second), was used to analyse agent interactions in successful and unsuccessful situations. Given that the sample performance situations studied had different durations, the respective time series trials needed to be normalized with respect to time for graphical comparison purposes.

Data were also subjected to quantitative analysis for measuring the variability within performance patterns. These selected methods included autocorrelation function (ACF) and approximate entropy (ApEn). The ACF assesses the dependency or stationarity of the distance gained time series (Borckardt, Nash, Murphy, Moore, Shaw, \& O'Neil, 2008; Derrick \& Thomas, 2004), being useful to appraise whether each distance gained data point is independent or unrelated to previous or succeeding observations (Borckardt et al., 2008). This technique was applied by plotting the Pearson productmoment correlation coefficient between data points at time $t$ and time $t+k-s$ lag for increasing values of $k$ (Wagenmakers, Farrell, \& Ratcliff, 2004). Accordingly, in order to understand how correlated the observations were with each other, and the scope of the long range dependency of the data, we computed autocorrelations for an array of 20 lags (.04-s lags ranging from .04 to .8) and plotted them in a correlogram to visually ascertain dependency. The ApEn method evaluates the complexity of time series data by quantifying the regularity or predictability within it (Pincus, 1991; Stergiou et al., 2004). According to Stergiou et al. (2004), in performance settings with a mixture of stochastic and deterministic systems, ApEn provides an effective statistical method. ApEn was considered appropriate for use since complex neurobiological systems are characterised by random, noisy deterministic and mixed data sets (Pincus, 1991). Due to inconsistent time series length, we used a normalized measure of ApEn, as suggested by Fonseca, Milho, Passos, Araújo, and Davids (submitted for publication) with input parameter $m=2$ and $r=.2$ (i.e., number of windows compared, tolerance factor, respectively) (Stergiou et al., 2004). For similar procedures see Passos, Araújo, \& Davids, et al. (2009).

Subsequently, the qualitative data were also subjected to statistical analysis in order to verify relationships with quantitative analyses. A non-parametric Mann-Whitney $U$ test was used to assess whether the two samples of performance situations (unsuccessful and successful phases-of-play) exhibited significant differences. Given that SPSS does not report an effect size index for the Mann-Whitney $U$ test, we have computed the size of the effect ( $r$ ) as $r=\mathrm{Z} / \sqrt{ } \mathrm{N}$ (c.f. Rosenthal \& DiMatteo, 2001).

\section{Results}

\section{Distance gained behaviour}

When plotting distance gained as a function of time, signs of stability in distance gained behaviours across time in successful situations can be observed (see exemplar data in Fig. 2a). In a successful attacking performance, distance gained seemed to demonstrate similar patterns over time. As in the exemplar successful attack displayed in Fig. 2A, in the entire sample (taking both successful and unsuccessful trials), this variable exhibited initially increasing, then decreasing negative values that approached zero. Since as successful attacks were considered those where the ball is carried beyond the gain line, in these attacks a zero crossover is observed. However, when comparing successful attacking outcomes against unsuccessful attacks, as displayed in Fig. 2b (exemplar trials), data curves although similar, achieved rather distinct values. This apparent trend in the performance data was observed over all trials and might be used to distinguish successful and unsuccessful patterns of attacking play.

An important observation is that the highest values for loss of territory (displayed by the maximum negative value of the series) seemed greater in unsuccessful attacking plays and occurred later in the time series data (see Fig. 2B).

\section{Maximum distance of retreat}

Maximum distance of retreat in each trial and the corresponding normalized value were verified next. The maximum distance of retreat for each performance situation seemed to be related to the time of occurrence in the two groups (see Fig. 3).

As illustrated in Fig. 3, successful and unsuccessful attacking plays formed distinct patterns which were clearly discriminated by the classification line plotted (LDA). The data showed that in successful plays the maximum distance of retreat (further back from the initial gain position) achieved lower values and occurred earlier than in unsuccessful attacks. The latter displayed greater retreating values and occurred later in the time series.

Statistical comparison revealed that the mean percentage time at which the maximum retreat distance was achieved significantly differed between successful and unsuccessful attacks $(Z=-2.61$, $p=.009, r=-.56)$, being higher in unsuccessful $(N=7 ; M=.40$; 

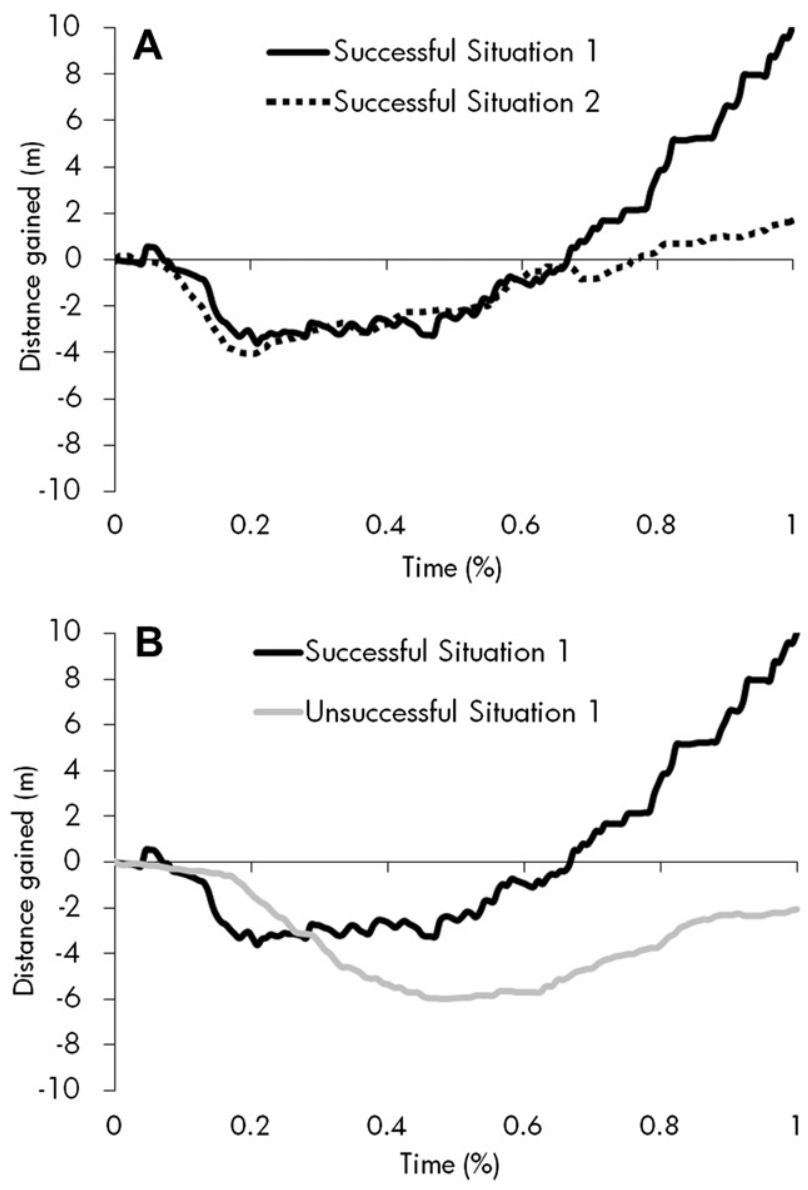

Fig. 2. Distance gained as function of normalized time for randomly selected exemplar data: (A) two illustrative successful trials; and (B) one illustrative unsuccessful and one illustrative successful trial.

$\mathrm{SD}=.12)$, compared to successful attacks $(N=15 ; M=.23$; $\mathrm{SD}=.10)$

Furthermore, the mean maximum retreat distance was shown to be significantly greater $(Z=-2.72, p=.007, r=-.58)$ for unsuccessful attacks $(N=7 ; M=-7.83 ; \mathrm{SD}=2.54)$, than for successful attacks $(N=15 ; M=-4.16 ; S=2.19)$.

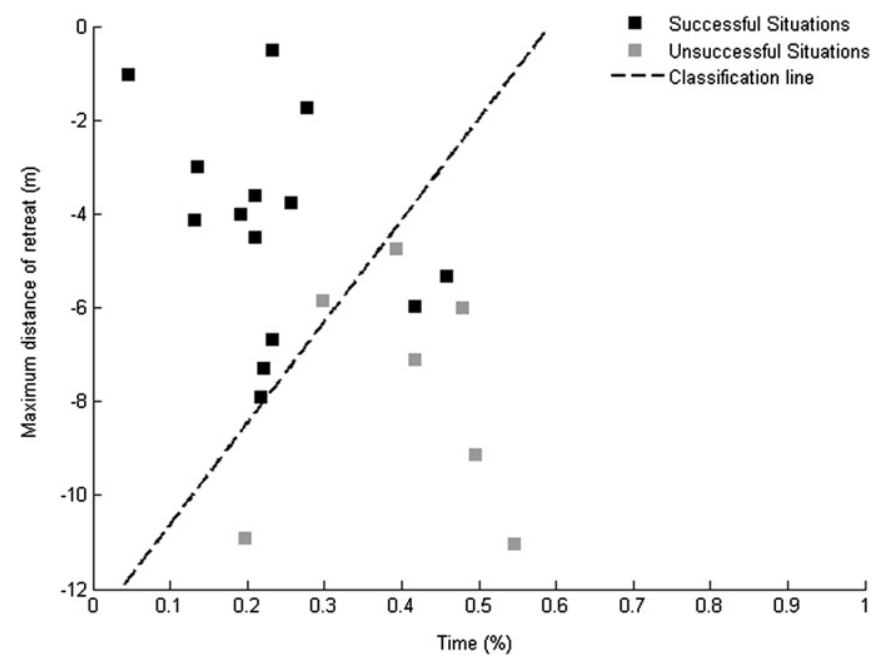

Fig. 3. Maximum distance of retreat achieved as a function of normalized time for the entire sample trials (square marks) and classification line (dashed line) from linear discriminant analysis (LDA).

\section{Territorial gain dynamics}

A phase-of-play is regarded as successful when it ends with the ball in a more advanced position in the field than the initial point of the attack. As a result, if the team in possession retreats in relation to its initial starting position, distance gained and its first derivate will be negative. When the ball is moved closer to the try line, the distance gained may be still negative (if ball doesn't cross gain line) or positive (once the gain line crossover occurs) and its first derivative values will be positive. Regarding the representation of distance gained behaviour in system state space (see Fig. 4), a phase plot of this variable as a function of its first derivate $D^{\prime}$ distinguished successful and unsuccessful attacks. Unsuccessful attacks showed more negative distance gained values, and more dispersed distance gained first derivate values. Successful attacks displayed more positive, but dispersed, distance gained values (i.e., the ball passed the initial starting position of the attack and was moved closer to the try line).

\section{Variability of distance gained time series}

In an attempt to understand the variability within the time series data, the autocorrelation method was applied to each distance gained time series. Results of this analysis suggested that observed behaviour over time tended to be highly correlated (Fig. 5, $0.6<r<1$ ), suggesting a long range dependence process unfolding in the data. The clear implication is that current behaviours were affected by prior behaviours in the same time series.

The correlogram produced from autocorrelation (Fig. 5) may be regarded as evidence for the stationarity of the time series, since correlation values obtained for each time lag decayed very slowly even with large values of the latter. Fig. 5 shows peak correlations at lags ranging from 1 to 20 data points, which indicate that even when the curve slides past a copy of itself, the fluctuations in the copy will line up with the original after being shifted, suggesting low levels of variability within these time series data.

In order to compare the complexity of this subsystem, we considered the ApEn $n_{\text {RatioRandom. For this normalized measure of }}$ entropy, values close to 1 indicate higher sub-system complexity and values close to 0 indicate more regularity. For successful

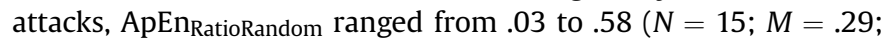
$\mathrm{SD}=.18$ ), whereas for unsuccessful attacks, these ranged from .3 to $.63(N=7 ; M=.46 ; \mathrm{SD}=.14)$. Such small values of ApEn $n_{\text {RatioRandom }}$ suggest a regular predictable nature of the time series data from

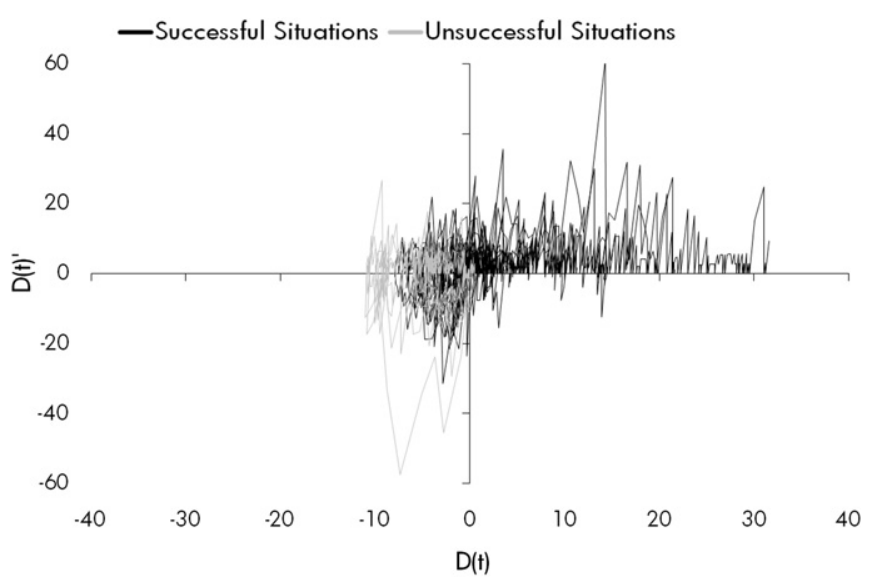

Fig. 4. Two-dimensional phase plot of the distance gained $D$ of the time series (on the horizontal axis) versus the first derivate $D^{\prime}$ (on the vertical axis) for the entire sample of successful and unsuccessful trials. 


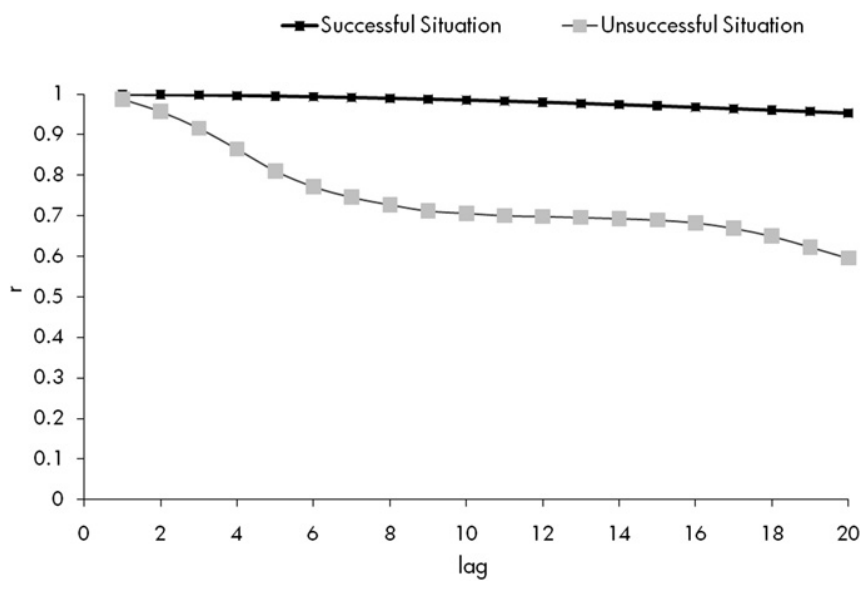

Fig. 5. Autocorrelation of distance gained time series for success and unsuccessful illustrative trials (.04-s lags).

attacking trials, regardless of the outcome. However, predictability was more noticeable in successful attacking sub-phases which presented a smaller mean value of ApEn $n_{\text {RatioRandom. }}$

We performed a non-parametric hypothesis test to determine if there were statistically significant differences between the ApEnRatioRandom for successful and unsuccessful attacks. Results revealed that unsuccessful trials displayed significantly higher entropy values $(Z=-2.01, p=.045, r=-.43)$.

\section{Discussion}

This study aimed to identify the collective dynamics of attack-defence rugby union phases-of-play preceding the possibility of a try being scored. Particularly, the focus was on the dynamics of territory gained in the playing field by the team in ball possession. An important challenge in studying complex pattern forming dynamics in field invasion games is to identify relevant collective or coordination variables (see e.g., McGarry et al., 2002). In field invasion games, it is important to advance up field when in possession of the ball to score a goal or try. Consequently, some studies of pattern forming dynamics in team sports have highlighted the centre of the team as an important variable to analyse (e.g., Bourbousson, Sève, \& McGarry, 2010b; Frencken \& Lemmink, 2008). In past work, this variable has been regarded as representing the forward and backward progression of each team on the field of play. In our study however, given the particular task constraints of rugby union with respect to the offside law ${ }^{1}$, distance gained was regarded as a suitable coordination variable for understanding structure in the second phases-of-play within this game. Coordination variables (known as order parameters or collective variables) and control parameters thought distinct are both relevant parameters and coimplicated in self-organized dynamical systems (Kelso, 1995). Nevertheless, as previously mentioned we didn't aim at finding the control parameters implicated in the coordinated systems of rugby phases-of-play. Instead, the data presented in this paper showed the evolution over time (dynamics) of a variable that captured the collective actions, i.e., the synergies or functional groupings of players functioning as a social entity (cf. Bourbousson et al., 2010b: Kelso, 2009; Marsh, Richardson, Baron, \& Schmidt, 2006). Our data demonstrated that distance gained was a variable capable of distinguishing between successful and unsuccessful attacks.

\footnotetext{
${ }^{1}$ According to IRB (2010) "In general play a player is offside if the player is in front of a team-mate who is carrying the ball, or in front of a team-mate who last played the ball." (p. 67).
}

Distance gained behaviour

Two different performance outcomes were categorized in this analysis: crossing or not crossing the gain line defined by ball's initial position. Time series analysis of successful attacks showed signs of consistency in distance gained dynamics in the different trials analyzed. Similarities in dynamics of distance gained behaviour indicated that, in successful or unsuccessful attacks, a cycle or sequence of cycles existed with the ball being moved backwards and then forwards (see exemplar data displayed in Fig. 2). This characteristic is in accordance with the description of rugby union as a team game of an intermittent character (Greenwood, 2003). However, instead of the 'stop and go' patterns of behaviour displayed by opposing players (Greenwood, 2003), our findings highlighted a more "backward and go" pattern.

\section{Phase-of-play dynamic behaviour distinguishing success in territorial gain}

Despite the similarities displayed in the dynamics of distance gained behaviour, this variable exhibited rather distinct values between successful and unsuccessful attacking plays. When the team in possession of the ball crossed the gain line, the pattern dynamics were characterised by different extents of backward and forward movements, compared to when the gain line was not crossed. The findings showed that successful attacks displayed lower values for maximum distance of ball retreat. Attacking teams tended to achieve those values sooner in the successful attacking phases, compared to unsuccessful phases when greater retreating values were recorded later in the time series (see Fig. 3). In concurrence with these results, Greenwood (2003) regarded rugby union as a team sport characterised by patterns of order and disorder. He also proposed that the longer the phase-of-play, the greater the probability of disorder and the difficulty in gaining territory in the field of play. In similar vein, studies of space-time patterns in other team sports have demonstrated moments of stability and instability over time within the particular subsystems of games analysed (e.g., Bourbousson, Sève, \& McGarry, 2010a, 2010b; Frencken \& Lemmink, 2008; McGarry, 2006).

Analysis of the dynamics of the maximum distance gained revealed that, although the backwards and forward movements are expected and common in any phase-of-play, the amplitude of those movements might be a distinguishing feature, indicative of a team's effectiveness in the attacking phase-of-play. This outcome was supported quantitatively by statistical analysis and was useful in categorizing successful and unsuccessful attacks. Successful and unsuccessful attacks were shown to be distinguished by the moment at which the peak distance of the ball's movement away from the gain line occurred (see Fig. 3).

The relations between the proposed variable and other related variables, such as its first derivate, supported the distinction between successful and unsuccessful phases-of-play based on the dynamics of territorial gain. A greater backward movement of the ball (more negative distance gained values) and velocities closer to zero tended to characterise unsuccessful attacks. Successful attacks displayed more positive values indicating that the ball had been moved ahead of the initial position towards the try line, with rather dispersed velocity values. Together distance gained and its derivatives revealed thus information on attacking success in rugby union, in terms of territorial gain.

\section{Variability of distance gained depicting success in territorial gain}

Distance gained values revealed, for both successful and unsuccessful attacks, high levels of dependency within the time 
series data (through autocorrelation analysis), suggesting a low level of system variability (i.e., evidence of stationarity within these data series). This long range dependency process unfolding in the data coincided with the complexity analysis carried out through

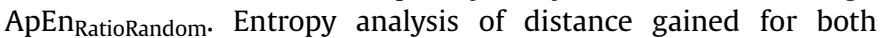
successful and unsuccessful attacks revealed a regular or predictability nature. The approximate entropy values of distance gained matched autocorrelation values, given that the regularity assumed by the former was consistent within series dependency verified in the latter. As several authors have stated, the amount of order/ disorder (chaos) within a system can be assessed by entropy measures (e.g., Sibella, Frosio, Schena, \& Borghese, 2007). Sibella et al. (2007), for instance, have investigated patterns in climbing strategies and used entropy (geometric entropy) as one of their performance measures. According to these authors, the low entropy values were observed for some climbers suggesting lower levels of energy expenditure, greater fluency, and a more effective climbing strategy. Entropy was, in this way, a measure of effectiveness of the movement. In our study, although low, statistically significant ApEn differences were verified between unsuccessful and successful attacks, with higher ApEn values observed in the former, evidence of less regularity and more complexity. Similar to the study of Sibella et al. (2007), ApEn values for each successful and unsuccessful attacking phase-of-play may also be a useful measure of performance effectiveness in team games.

\section{Collective behaviour conveyed by distance gained dynamics}

Regardless of the small sample of match situations studied and depth of our analysis, we may extrapolate some performance trends of distance gained dynamics that may allow prediction of the success or failure of phases-of-play in the team sport of rugby union. The present findings were consistent with Greenwood's (2003) proposal that, achieving a more advanced position in the field of play must be regarded as one of the key attacking principles in rugby union performance. He considered that this goal could be achieved either by the team in possession of the ball, or by the defending team by forcing the former to retreat. The emphasis in this study was the location and pace of ball displacement as a marker of successful territorial occupation. The achievement of a more advanced position in the field can be simply understood as successful performance and can be evaluated by the degree of territorial gain by assessing the position reached with the ball and its behaviour over time, i.e., its dynamics. Other studies have also shown that the analysis of the dynamics of a single variable could reflect the global dynamics of an entire neurobiological system (e.g., Vereijken et al., 1997) and of the dyadic interactions between team games players (e.g., Passos et al., 2008). Previous studies on the dynamics of decision making behaviours in sport have been mainly focused on team sports, typically in 1vs1 interactions (e.g., Araújo, Davids, Bennett, Button, \& Chapman, 2004; Davids et al., 2006), or in dyadic sports (e.g., squash by McGarry et al., 2002). This study advanced understanding by investigating interactions of multi-player sub-phases in field invasion team games. The findings supported previous research (e.g., Marsh et al., 2006; Bourbousson et al., 2010b) showing how two or more individuals may function as one entity. For instance Marsh et al. (2006) defined a "collective" as an emergent "social unit of action in which individual actors become part of a larger social entity, a collective, because it characterises social interactions in their most abstract and basic form" (p.4). Our findings considered that players in a team interact with each other while cooperating in a goal-directed manner and that a team may be assumed a single entity or collective. Distance gained can be regarded as a coordination or collective variable capturing and describing the collective interactions between two teams in a match, revealing features that distinguish success of an attack during a phase-of-play.

Sustained on the ecological dynamics approach (Araújo et al., 2006) data from the present study supported the choice of distance gained as a variable which illustrates the dynamics of successful and unsuccessful second phases-of-play in rugby union. Analysis of ball displacement trajectory patterns (used to compute evolving distance gained) did reveal distinct behaviour in successful and unsuccessful attacks.

As mentioned before, the back and forward pattern displayed by this variable might be related with the task constraint of backward pass. That is, forward movement patterns observed in the game might be hampered by this rule. In this manner, this task constraint might work thus as meaningful information in the extent that influences the emergent game patterns. Conversely, the dynamics of the backwards or forward movement displayed by the ball may also influence how that constraint can be exploited. This circular or reciprocal relation between a rule of the game and the behaviour patterns might be worth of investigation in a further study.

Furthermore, the methodology used in this study proved useful in involving many social neurobiological system elements (such as each player's movement dynamics, referees, etc.). Although distance gained has been suggested as a coordination variable in this study, further work is needed to identify potential control parameters that might be influential in this context. To empirically and unambiguously verify the existence of a collective or coordination variable, qualitative changes must be identified caused by variation in the control parameter (cf. Kelso, 1995, 2009). In summary, this study showed that distance gained dynamics manifests a characteristic collective behaviour pattern that potentially captures the macroscopic order of a multi-player attack-defence sub-phase in the team sport of rugby union, depicting successful and unsuccessful patterns of play.

\section{Acknowledgments}

This work was funded by a project grant SFRH/BD/36480/2007 awarded by the Foundation for Science and Technology (Portugal) to the first author.

\section{References}

Araújo, D., Davids, K., Bennett, S., Button, C., \& Chapman, G. (2004). Emergence of sport skills under constraints. In A. M. Williams, \& N. J. Hodges (Eds.), Skill acquisition in sport: Research, theory and practice (pp. 409-433). London: Routledge, Taylor \& Francis.

Araújo, D., Davids, K., \& Hristovski, R. (2006). The ecological dynamics of decision making in sport. Psychology of Sport and Exercise, 7(6), 653-676.

Best, J. P., McIntosh, A. S., \& Savage, T. N. (2005). Rugby World Cup 2003 injury surveillance project. British Journal of Sports Medicine, 39, 812-817.

Bessa, J. P. (2005). Da cultura táctica à tomada de decisão num jogo colectivo de combate. In D. Araújo (Ed.), O contexto da decisão (pp. 253-258). Lisboa: Visão e contextos.

Borckardt, J. J., Nash, M. R., Murphy, M. D., Moore, M., Shaw, D., \& O'Neil, P. (2008) Clinical practice as natural laboratory for psychotherapy research: a guide to case-based time-series analysis. American Psychologist, 63(2), 77-95.

Bourbousson, J., Sève, C., \& McGarry, T. (2010a). Space-time coordination dynamics in basketball: part 1. Intra- and inter-couplings among player dyads. Journal of Sports Sciences, 28(3), 339-347.

Bourbousson, J., Sève, C., \& McGarry, T. (2010b). Space-time coordination dynamics in basketball: part 2. The interaction between the two teams. Journal of Sports Sciences, 28(3), 349-358.

Davids, K., Button, C., Araújo, D., Renshaw, I., \& Hristovski, R. (2006). Movement models from sports provide representative task constraints for studying adaptive behavior in human motor systems. Adaptive Behavior, 14, 73-95.

Davids, K., Araújo, D., Shuttleworth, R., \& Button, C. (2003). Acquiring Skill in Sport: a constraints-led perspective. International Journal of Computer Science in Sport, 2(2), 31-39.

Derrick, T. R., \& Thomas, J. M. (2004). Time series analysis: the cross-correlation function. In N. Stergiou (Ed.), Innovative analyses of human movement (pp. 189-205). Champaign, Illinois: Human Kinetics Publishers. 
Duarte, R., Araújo, D., Fernandes, O., Fonseca, C., Correia, V., Gazimba, V., et al (2010). Capturing complex human behaviors in representative sports contexts with a single camera. Medicina (Kaunas), 46(6), 408-414.

Fernandes, O., Folgado, H., Duarte, R., \& Malta, P. (2010). Validation of the tool for applied and contextual time-series observation. International Journal of Sport Psychology, 41(4), 63-64.

Fonseca, S., Milho, J., Passos, P., Araújo, D., \& Davids, K. (submitted for publication). Validity of approximate entropy normalized measures for analyzing inter-agent coordination in social neurobiological systems. Behaviour Research Methods.

Frencken, W. G. P., \& Lemmink, K. A. P. M. (2008). Team kinematics of small-sided soccer games: a systematic approach. In T. Reilly, \& F. Korkusuz (Eds.), Science and football VI: Proceedings of the 6th world congress on science and football (pp. 161-166). London: Routledge.

Greenwood, J. (2003). Total rugby. Fifteen-man rugby for coach and player ( $5^{\text {th }}$ ed.). London: A \& C Black (Publishers) Ltd

International Rugby Board. (2010). Laws of the game rugby union 2010. Retrieved February 9, 2010, from. http://www.irblaws.com/downloads/EN/irb_law_book_ 2010_en.pdf.

Keil, D., Holmes, P., Bennett, S., Davids, K., \& Smith, N. (2000). Theory and practice in sport psychology and motor behaviour needs to be constrained by integrative modelling of brain and behaviour. Journal of Sports Sciences, 18(6), 433-443.

Kelso, J. A. S. (1995). Dynamic patterns. The self-organization of brain and behavior. Cambridge, MA: The MIT Press.

Kelso, J. A. S. (2002). The complementary nature of coordination dynamics: selforganization and agency. Nonlinear Phenomena in Complex Systems, 5(4), 364-371.

Kelso, J. A. S. (2009). Coordination dynamics. In R. A. Meyers (Ed.), Encyclopedia of complexity and system science (pp. 1537-1564). Heidelberg: Springer.

Marsh, K. L., Richardson, M. J., Baron, R. M., \& Schmidt, R. C. (2006). Contrasting approaches to perceiving and acting with others. Ecological Psychology, 18 $1-38$.

McGarry, T. (2006). Identifying patterns in squash contests using dynamical analysis and human perception. International Journal of Performance Analysis in Sport, 6 , 134-147.

McGarry, T., Anderson, D., Wallace, S., Hughes, M., \& Franks, I. (2002). Sport Competition as a dynamical self-organizing system. Journal of Sport Sciences, 20(10), 771-781.

Mullineaux, D. R. (2008). Research methods: sample size and variability effects on statistical power. In C. J. Payton, \& R. M. Bartlett (Eds.), Biomechanical evaluation of exercise movement in sport and exercise. The British association of sport and exercise sciences guidelines (pp. 153-175). London: Routledge, Taylor \& Francis.

Mullineaux, D. R., Bartlett, R. M., \& Bennett, S. (2001). Research design and statistics in biomechanics and motor control. Journal of Sports Sciences, 19(10), 739-760.

Neisser, U. (1994). Multiple systems: a new approach to cognitive theory. European Journal of Cognitive Psychology, 6, 225-241.

Newell, K. M. (1986). Constraints on the development of coordination. In M. G. Wade, \& H. T. A. Whiting (Eds.), Motor Development in Children: Aspects of Coordination and Control (pp. 341-359). Boston, MA: Martinus Nijhop.

Passos, P., Araújo, D., Davids, K., Gouveia, L., Serpa, S., Milho, J., et al. (2009). Interpersonal pattern dynamics and adaptive behavior in multiagent neurobiological systems: conceptual model and data. Journal of Motor Behavior, 41(5), 445-459.

Passos, P., Araújo, D., Davids, K., \& Shuttleworth, R. (2008). Manipulating constraints to train decision making in rugby union. International Journal of Sports Science $\mathcal{E}$ Coaching, 3(1), 125-140.

Pincus, S. M. (1991). Approximate entropy as a measure of system complexity. Proceedings of the National Academy of Sciences, 88, 2297-2301.

Reed, D., \& Hughes, M. (2006). An exploration of team sport as a dynamical system. International Journal of Performance Analysis in Sport, 6(2), 114-125.

Rosenthal, R., \& DiMatteo, M. R. (2001). Meta-analysis: recent developments in quantitative methods for literature reviews. Annual Review of Psychology, 52, 59-82.

Schweitzer, F. (1997). Self-organization of complex structures. From individual to collective dynamics. CRC Press.

Sibella, F., Frosio, I., Schena, F., \& Borghese, N. A. (2007). 3D Analysis of the body center of mass in rock climbing. Human Movement Science, 26, 841-852.

Stergiou, N., Buzzi, U. H., Kurz, M. J., \& Heidel, J. (2004). Nonlinear tools in human movement. In $\mathrm{N}$. Stergiou (Ed.), Innovative analyses of human movement (pp. 63-92). Champaign, Illinois: Human Kinetics Publishers.

van Gelder, T. J., \& Port, R. (1995). It's about time: an overview of the dynamical approach to cognition. In R. Port, \& T. J. van Gelder (Eds.), Mind as motion: Explorations in the dynamics of cognition (pp. 1-43). Cambridge MA: MIT Press.

Vereijken, B., van Emmerik, R. E. A., Bongaardt, R., Beek, W. J., \& Newell, K. M. (1997). Changing coordinative structures in complex skill acquisition. Human Movement Science, 16, 823-844.

Wagenmakers, E.-J., Farrell, S., \& Ratcliff, R. (2004). Estimation and interpretation of 1/fa noise in human cognition. Psychonomic Bulletin E' Review, 11(4), 579-615. 